\title{
Genetic variation and expression levels of tight junction genes identifies association between MAGI3 and inflammatory bowel disease
}

Elisabeth Norén ${ }^{1,2^{*}}$, Sven Almer ${ }^{1,3}$ and Jan Söderman ${ }^{2,4}$

\begin{abstract}
Background: Inflammatory bowel disease (IBD) is associated with increased intestinal permeability, which involves paracellular passage regulated through tight junctions (TJ). The aim of the study was to investigate single nucleotide polymorphisms (SNP) located in genes encoding interacting TJ proteins and corresponding expressions, in relation to IBD.

Methods: Allelic associations between TJ-related genes (F11R, MAGI1, MAGI2, MAGI3, PARD3, PTEN, and TJP1) and IBD, Crohn's disease (CD), or ulcerative colitis (UC) were investigated. PTPN22 was included since it's located in the same genetic region as MAG/3. Gene expression levels were investigated in relation to genotype, inflammatory status, phenotype, and medical treatment.

Results: The two strongest allelic associations were observed between IBD and SNPs in MAGI2 (rs6962966) and MAG/3 (rs1343126). Another MAGI3 SNP marker (rs6689879) contributed to increased ileal MAG/3 expression level in non-IBD controls. Furthermore, association between inflammation and decreased expression levels of MAG/3, PTEN, and TJP1 in colonic IBD as well as UC mucosa, and between inflammation and increased expression of PTPN22 in colonic IBD mucosa, was observed.
\end{abstract}

Conclusions: Our findings lend support to a genetic basis for modulation of intestinal epithelial barrier in IBD, and we have identified MAG/3 as a new candidate gene for IBD.

Keywords: Inflammatory bowel disease, Tight junctions, Single nucleotide polymorphism, Genetic predisposition, Gene expression

\section{Background}

Inflammatory bowel disease (IBD), including Crohn's disease (CD) and ulcerative colitis (UC), are complex diseases thought to result from loss of homeostasis between the intestinal microbial milieu, the immune system, and a genetic predisposition [1]. Genetic association in IBD has been the focus of much research [2,3] leading to the recent identification of a large number of susceptibility loci [3-5]. Still, the hitherto identified genetic

* Correspondence: elisabeth.noren@rjl.se

${ }^{1}$ Department of Medicine, Karolinska Institutet, Solna, Stockholm, Sweden

${ }^{2}$ Division of Medical Diagnostics, Region Jönköping County, Jönköping, Sweden

Full list of author information is available at the end of the article associations only account for a small fraction of the heritability of CD and UC [3].

IBD has been linked to increased paracellular permeability [6, 7]. The intestinal permeability is further increased in both $C D$ patients and their relatives, indicating underlying hereditary factors [8]. However, it remains controversial whether this affected permeability is primary, caused by genetic factors, and/or secondary to inflammation or environmental factors. The tight junction (TJ) structure is critical for the permeability properties of the intestine [9] and several studies provide support for a genetic basis in increased permeability [10].

Wapenaar et al. [11] have described an association between celiac disease and single nucleotide polymorphism 
(SNP) markers in MAGI2 and PARD3. This MAGI2 marker was also associated with UC [11]. Additionally, McGovern et al. [12] identified a link between genetic variation in MAGI2 and both $\mathrm{CD}$ and $\mathrm{UC}$.

The aim of this study was to investigate relations between IBD and MAGI2 and PARD3, as well as other TJ genes (F11R, MAGI1, MAGI3, PTEN, and TJP1) encoding products interacting with each other (Fig. 1) [1315]. Additionally, PTPN22 was included since it is located in the same genetic region as MAGI3 (Fig. 2) and has previously been described in relation to IBD [5, 16]. To gain a better understanding of the pathogenic mechanism of IBD we further analyzed ileal and colonic gene expression in relation to genotype, inflammatory status, phenotype, and ongoing medical treatment.

\section{Methods}

\section{Study subjects}

Genetic association was investigated in adult Swedish patients with IBD (138 and 157 patients with CD or UC, respectively) and controls from an anonymized regional DNA bank consisting of randomly selected individuals $(n=423)$ living in the southeastern part of Sweden was used after verbal permission from Prof. Peter Söderkvist (Department of Clinical and Experimental Medicine, Linköping University, Linköping, Sweden) (Table 1, subgroup 1).

A second Swedish cohort (Table 2, subgroup 2), from which both DNA and RNA were available, was recruited to follow up the case-control study of subgroup 1 . Blood samples and intestinal biopsy specimens were obtained from adult IBD patients and non-IBD controls. Each intestinal biopsy was categorized as inflamed or non-inflamed based on a compound evaluation of endoscopic findings assessed by one experienced endoscopist (S.A.) and routine histopathologic assessment for inflammation. Only biopsies with concordant results were

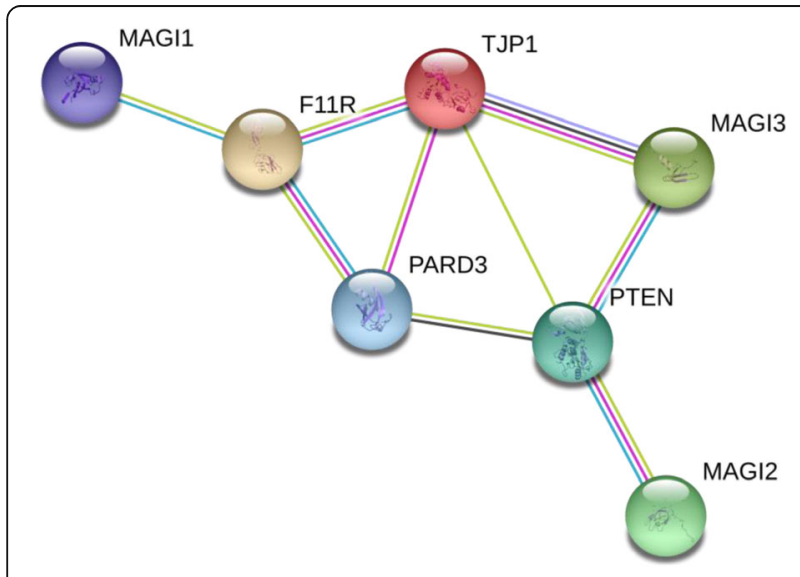

Fig 1 A network consisting of seven TJ genes (F11R, MAG/1, MAG/2, MAG/3, PARD3, PTEN, and TJP1) identified using the STRING search tool [14, 15] analyzed further. In total the study included biopsies from 52 Swedish IBD patients (42 inflamed biopsies and 55 non-inflamed biopsies), including $21 \mathrm{CD}$ patients (16 inflamed biopsies and 24 non-inflamed biopsies), $29 \mathrm{UC}$ patients (24 inflamed biopsies and 29 non-inflamed biopsies), 2 IBD-type unclassified (IBDU; 2 inflamed biopsies and 2 non-inflamed biopsies), and 33 non-inflamed non-IBD controls (86 biopsies).

\section{SNP selection for genetic association studies}

TJ-related genes (F11R, MAGI1, MAGI2, MAGI3, PARD3, PTEN, and TJP1) encoding products interacting with each other $[13,14]$ were investigated (Fig. 1). Additionally, PTPN22 was included since it is located in the same genetic region as MAGI3 (Fig. 2) and has previously been described in relation to $\operatorname{IBD}[5,16]$. All SNP markers are given in Additional file 1: Table S1.

SNP markers (minor allele frequency $\geq 10 \%$, pair-wise $\left.r^{2} \geq 0.8\right)$ of F11R, MAGI1, MAGI3, and PTEN were selected using SNPbrowser Software version 4.0 (Applied Biosystems, Foster City, CA). SNP markers for MAGI1 and MAGI3 were limited to exons and exon-intron boundaries, due to the large size of these genes. All SNP markers in the genetic region of MAGI2 [11, 12], MAGI3-PTPN22 [5], PARD3 [11], TJP1 [11], and an additional marker for MAGI1 (rs9880851) [11] were selected from the literature.

\section{Definition of genetic blocks of MAGI3 and PTPN22}

Blocks of genetic linkage disequilibrium in the region of MAGI3 and PTPN22 were identified using data from HapMap (available at: www.hapmap.org; HapMap Data Rel. 28 Phase II + III, August10) (Fig. 2). The blocks were visualized using Haploview and the algorithm by Gabriel et al. [17] based on 95\% confidence bounds on D' (Haploview version 4.2, available at: www.broad.mit.edu/haploview).

\section{Genotyping}

DNA was isolated from buffy coat or whole blood (EDTA blood) using the MagNA Pure LC DNA Isolation Kit and MagNA Pure extraction robot (Roche, Basel, Switzerland). In patients where no DNA from blood was available, the genotyping was performed using DNA isolated from intestinal biopsies (isolation previously described [18]). Allelic discrimination was carried out using either the TaqMan OpenArray system or real-time PCR, as well as TaqMan SNP genotyping assays (Applied Biosystems, Foster City, CA) (Additional file 1: Table S1).

Allelic discrimination using TaqMan OpenArray Genotyping Plates, TaqMan OpenArray Genotyping Master Mix, GeneAmp PCR System 9700, and OpenArray NT Imager (Applied Biosystems) was in accordance with the manufacturer's recommendation. Genotype data were 


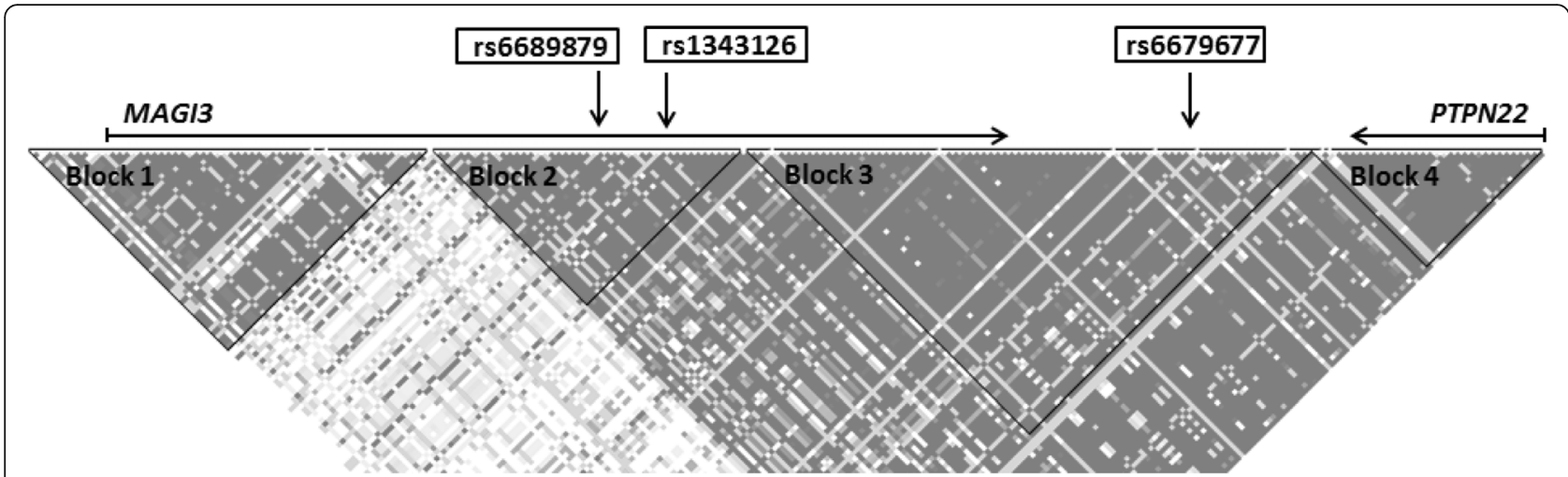

Fig 2 Illustration of the genetic region of MAG/3 and PTPN22. SNP data were obtained from the HapMap database (available at: www.hapmap.org; HapMap Data Rel 28 Phase II + III, August10) and analyzed using Haploview version 4.2 (available at www.broad.mit.edu/haploview) with blocks defined according to Gabriel et al. [17]

analyzed using OpenArray SNP Genotyping Analysis Software version 1.0.3 and TaqMan Genotyper Software v.1.3 (Applied Biosystems).

The allelic discrimination using real-time PCR was performed in a total reaction volume of $10 \mu \mathrm{L}$, consisting of TaqMan SNP genotyping assays and TaqMan Genotyping Master Mix using either the 7500 Fast RealTime PCR System (Applied Biosystems) or the CFX96 Real-time System, C1000 Touch Thermal Cycler (BioRad Laboratories Inc., Hercules, CA). The genotype data was analyzed using 7500 Software version 2.0.6 (Applied Biosystems) or Bio-Rad CFX Manager 3.1 (Bio-Rad Laboratories Inc.).

\section{Gene expression analysis}

For genes with a significant genetic association to IBD, $\mathrm{CD}$, or UC (in this study), gene expression was analyzed in relation to genotype, inflammatory status, phenotype, and ongoing medical treatment.

RNA purification was performed as described previously [18]. cDNA was synthesized from $2 \mu \mathrm{g}$ RNA in a total volume of $40 \mu \mathrm{L}$ using High Capacity cDNA Reverse Transcription kit with RNase Inhibitor according to the manufacturer's instructions (Invitrogen, Carlsbad, $\mathrm{CA}$ ). The cDNA was diluted to $25 \mathrm{ng} / \mu \mathrm{L}$ using $0.1 \times \mathrm{TE}$ buffer and stored at $-80{ }^{\circ} \mathrm{C}$ until analysis.

Gene expression of F11R (Hs00170991_m1), MAGI2 (Hs00202321_m1), MAGI3 (Hs00326365_m1), PTEN

Table 1 Summary of study participants in subgroup 1

\begin{tabular}{lll}
\hline Cohort and disease $^{\mathrm{a}}$ & Number of individuals & Number of women \\
\hline Control subjects & 423 & $223(52.7 \%)$ \\
CD cases & 138 & $72(52.2 \%)$ \\
UC cases & 157 & $70(44.6 \%)$ \\
\hline
\end{tabular}

$C D$ Crohn's disease, UC ulcerative colitis

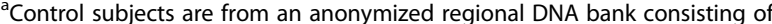
randomly selected individuals living in the southeastern part of Sweden
(Hs02621230_s1), PTPN22 (Hs01587518_m1), and TJP1 (Hs01551861_m1) was analyzed using TaqMan Gene Expression Assay (Applied Biosystems), TaqMan Universal Mastermix (Applied Biosystems), and a 7500 Fast RealTime PCR System (Applied Biosystems). Each individual reaction contained $10 \mathrm{ng} \mathrm{cDNA}$ in a total reaction volume of $20 \mu \mathrm{L}$.

Threshold cycle $\left(\mathrm{C}_{\mathrm{T}}\right)$ values were established (ExpressionSuite Software Version 1.0.3; Applied Biosystems) and normalized to the average of selected reference genes (CASC3 (Hs00201226_m1), UBA52 (Hs03004332_g1), and POP4 (Hs00198357_m1) [18]) generating delta- $\mathrm{C}_{\mathrm{T}}$ $\left(\Delta C_{\mathrm{T}}\right)$ values. Relative quantification $(\mathrm{RQ})$ values were further established, according to Livak et al. [19], by relating the $\Delta C_{T}$ value to the sample with the lowest gene expression for each gene (using Microsoft Office Excel;

Table 2 Summary of study participants in subgroup 2

\begin{tabular}{|c|c|c|c|}
\hline $\begin{array}{l}\text { Disease and } \\
\text { subgroups }\end{array}$ & $\begin{array}{l}\text { Number of } \\
\text { individuals } \\
\text { (women) }\end{array}$ & $\begin{array}{l}\text { Number of non- } \\
\text { inflamed biopsies }\end{array}$ & $\begin{array}{l}\text { Number of } \\
\text { inflamed } \\
\text { biopsies }\end{array}$ \\
\hline $\mathrm{IBD}$ & $52(29)$ & & \\
\hline Ileum & & 24 & 7 \\
\hline Colon & & 31 & 35 \\
\hline$C D$ & $21(13)$ & & \\
\hline Ileum & & 11 & 7 \\
\hline Colon & & 13 & 9 \\
\hline UC & $29(15)$ & & \\
\hline Ileum & & 12 & 0 \\
\hline Colon & & 17 & 24 \\
\hline Non-IBD & $33(20)$ & & \\
\hline Ileum & & 24 & 0 \\
\hline Colon & & 62 & 0 \\
\hline
\end{tabular}

IBD inflammatory bowel disease, CD Crohn's disease, UC ulcerative colitis, IBDU IBD-type unclassified, non-IBD non-inflamed non-IBD controls

${ }^{a}$ The total IBD group included CD $(n=21)$, UC $(n=29)$, and IBDU $(n=2)$ patients 
Microsoft Corporation, Redmond, WA). Group differences in gene expression were calculated as a fold change (fc) based on RQ values.

\section{Statistical analysis}

Allelic odds ratios (OR) and $p$-values, based on chisquared $(\chi 2)$ tests, were calculated using JMP Genomics 6.0 (JMP Genomics 6.0; SAS Institute Inc., Cary, NC). Deviation from Hardy-Weinberg equilibrium was analyzed using the exact test implemented in Haploview version 4.2 (available at: http://www.broad.mit.edu/hap loview). For these statistical tests, $p<0.05$ was considered significant.

Genes with significant association to IBD, CD, or UC were further analyzed. Group differences in gene expression were investigated using Kruskal-Wallis ANOVA or Mann-Whitney $U$ test (Statistica 12; StatSoft Inc., Tulsa, OK). Gene expression levels were investigated in relation to genotype using Spearman's rank correlation test (Statistica version 12.7; StatSoft Inc., Tulsa, OK) and also in relation inflammatory status, phenotype, and ongoing medical treatment using logistic regression (Statistica version 12.7; StatSoft Inc.). The analyses of the group differences of gene expressions as well as the logistic regressions were performed using $\Delta \mathrm{C}_{\mathrm{T}}$ values. Box plots were created using Statistica version 12.7 (StatSoft Inc.). A Bonferroni adjusted $p<0.008$ (based on the number of analyzed genes; $n=6$ ) was considered significant for statistical analysis based on gene expression levels.

\section{Ethical considerations}

This study was approved by the ethics committee of Linköping University (dnr. M35-07, dnr. 2011/201-31). Written and informed consent were obtained from all study participants.

\section{Results}

Genetic association - case-control approach

Of the 64 SNP markers, twelve were excluded due to failed genotyping or absence of Hardy-Weinberg equilibrium (Additional file 1: Table S1). The two strongest associations were observed between IBD overall and SNP markers in MAGI2 (rs6962966; susceptibility allele A; $p=$ 0.004 ) and MAGI3 (rs1343126; susceptibility allele T; $p=$ 0.004) (Additional file 2: Table S2). These two markers were also associated with $\mathrm{CD}(p=0.008$ and $p=0.051$, respectively) and UC ( $p=0.045$ and $p=0.011$, respectively) individually, even if the association between $\mathrm{CD}$ and the MAGI3 SNP marker was borderline significant. Associations were also observed between IBD overall and MAGI3 (rs12119076, $p=0.022$ ), as well as between UC and F11R (rs7546890, $p=0.043)$, MAGI2 (rs7803276, $p=0.031$ ), MAGI3 (rs6689879, $p=0.043$ ), PTEN (rs1234226, $p=$ 0.046), and TJP1 (rs260526, $p=0.010$ ).

\section{Gene expression}

Biopsies from different colonic segments (cecum, ascending colon, transverse colon, descending colon, sigmoid colon, and rectum) were treated as biological replicates since all genes except TJP1 were expressed at

Table 3 For genes with a significant genetic association to IBD, CD, or UC, gene expression ( $\Delta C$ t values) was analyzed in relation to genotype, in intestinal biopsies from the different subgroups, using Spearman's rank correlation test

\begin{tabular}{|c|c|c|c|c|c|c|c|}
\hline \multirow{3}{*}{ Gene } & \multirow{3}{*}{ Gene SNP ${ }^{a}$} & \multicolumn{2}{|l|}{ Non-IBD } & \multicolumn{2}{|c|}{ Non-inflamed IBD } & \multicolumn{2}{|l|}{ Inflamed IBD } \\
\hline & & Ileum & Colon & lleum & Colon & Ileum & Colon \\
\hline & & $p$-value ${ }^{b}$ & $p$-value ${ }^{b}$ & $p$-value ${ }^{b}$ & $p$-value ${ }^{b}$ & $p$-value ${ }^{b}$ & $p$-value ${ }^{b}$ \\
\hline$\overline{F 11 R}$ & rs7546890 (T) & $0.144(5,14,5)$ & $0.112(6,19,8)$ & $0.167(5,13,6)$ & $0.541(8,15,6)$ & $0.140(1,4,1)$ & $0.347(7,16,5)$ \\
\hline \multirow[t]{2}{*}{$M A G / 2$} & rs7803276 (C) & $0.009(10,8,6)$ & $0.603(11,15,7)$ & $0.604(5,11,8)$ & $0.029(8,14,7)$ & $0.637(3,2,1)$ & $0.285(6,13,9)$ \\
\hline & rs6962966 (A) & $0.797(11,8,5)$ & $0.657(14,11,8)$ & $0.266(5,11,8)$ & $0.868(6,16,7)$ & $0.954(3,1,2)$ & $0.817(6,13,9)$ \\
\hline \multirow[t]{4}{*}{$M A G / 3$} & rs6689879 (C) & $0.002(4,7,13)$ & $0.050(5,10,18)$ & $0.497(1,8,15)$ & $0.785(2,13,14)$ & $0.021(0,3,3)$ & $0.355(1,13,14)$ \\
\hline & rs1343126 (T) & $1.000(0,14,10)$ & $0.916(0,16,17)$ & $0.044(1,15,8)$ & $0.737(2,14,13)$ & $0.594(1,3,2)$ & $0.780(0,18,10)$ \\
\hline & rs12119076 (C) & $0.978(0,13,11)$ & $0.777(0,15,18)$ & $0.044(1,15,8)$ & $0.737(2,14,13)$ & $0.594(1,3,2)$ & $0.780(0,18,10)$ \\
\hline & rs $6679677^{d}$ & $0.266(18,6,0)$ & $0.018(25,8,0)$ & $0.477(22,2,0)$ & $0.834(26,3,0)$ & $N A^{c}(6,0,0)$ & $0.047(25,3,0)$ \\
\hline PTEN & rs1234224 (G) & $0.554(2,12,10)$ & $0.458(3,16,14)$ & $0.851(1,9,14)$ & $0.772(2,15,12)$ & $N A^{c}(0,0,6)$ & $0.695(3,14,11)$ \\
\hline \multirow[t]{4}{*}{ PTPN22 } & rs6689879 (C) & $0.697(4,7,13)$ & $0.509(5,10,18)$ & $0.634(1,8,15)$ & $0.129(2,13,14)$ & $0.854(0,3,3)$ & $0.764(1,13,14)$ \\
\hline & rs1343126 (T) & $0.227(0,14,10)$ & $0.025(0,16,17)$ & $0.870(1,15,8)$ & $0.822(2,14,13)$ & $0.908(1,3,2)$ & $0.963(0,18,10)$ \\
\hline & rs12119076 (C) & $0.555(0,13,11)$ & $0.089(0,15,18)$ & $0.870(1,15,8)$ & $0.822(2,14,13)$ & $0.908(1,3,2)$ & $0.963(0,18,10)$ \\
\hline & rs6679677 & $0.128(18,6,0)$ & $0.183(25,8,0)$ & $0.147(22,2,0)$ & $0.291(26,3,0)$ & $N A^{c}(6,0,0)$ & $0.745(25,3,0)$ \\
\hline TJP1 & rs260526 (G) & $0.285(21,3,0)$ & $0.052(29,4,0)$ & $0.582(21,3,0)$ & $0.396(24,5,0)$ & $N A^{c}(6,0,0)$ & $0.517(22,5,1)$ \\
\hline
\end{tabular}

IBD inflammatory bowel disease, CD Crohn's disease, UC ulcerative colitis

${ }^{a}$ The susceptibility allele is shown in parentheses. ${ }^{b}$ Number of individuals in each genotype group is shown in parentheses (individuals homozygous for the susceptibility allele, heterozygous individuals, and individuals homozygous for the non-susceptibility allele). ${ }^{\mathrm{C}} \mathrm{NA}$; not applicable. ${ }^{\mathrm{d}}$ The susceptibility allele (C) is defined from Jostins et. al [5] 
equal levels at these locations in non-IBD controls (data not shown). TJP1 showed slightly higher expression in the sigmoid colon compared to ascending colon $(p=$ 0.006 ; $\mathrm{fc}=1.16$ ). The biopsy specimens from ileum and colon were analyzed separately.

\section{Gene expression versus genotype}

Carriage of at least one UC susceptibility allele (C) of MAGI3 rs6689879 contributed to increased MAGI3 expression level in ileal non-IBD mucosa $(p=0.002$; $\mathrm{fc}=$ 1.7) (Table 3). No other SNP markers contributed significantly to the gene expression level of corresponding genes.

\section{Gene expression versus inflammation}

Biopsies from inflamed colonic IBD and UC mucosa expressed significant lower levels of MAGI3, PTEN, and TJP1, compared to non-inflamed colonic mucosa from the same groups of patients (Table 4). There was no overlap between the expression levels of PTEN in inflamed colonic mucosa of $\mathrm{CD}$ patients compared to non-inflamed CD mucosa (Fig. 3). Furthermore, biopsies from inflamed colonic IBD mucosa expressed a significantly higher level of PTPN22, compared to noninflamed colonic IBD mucosa.

\section{Gene expression versus phenotype}

The gene expression levels of analyzed genes were not different between any of the phenotypes (CD, UC, or non-IBD) (Additional file 3: Table S3).

\section{Gene expression versus medication}

Expression of investigated genes in non-inflamed and inflamed IBD biopsy specimens was not affected by ongoing medical treatment (thiopurines, aminosalicylates, or glucocorticosteroids) (Additional file 4: Table S4). Too few individuals underwent anti-TNF- $\alpha$-antibody treatment for meaningful statistical analysis.

\section{Discussion}

IBD has been associated with an increased paracellular permeability of the intestinal epithelium and a dysfunctional barrier is considered important in the pathogenesis of IBD [6, 7]. The TJ structure is critical for the paracellular barrier properties [9]. Although a few IBD susceptibility markers have been linked to barrier related genes, e.g. CLDN2 and MAGI2 [11, 12, 20], the extent to which a dysfunctional barrier is a consequence of genetic factors remains unclear.

The large numbers of susceptibility loci identified in relation to IBD [3-5] only explain $10.9 \%$ and $7.7 \%$ of the heritability of $\mathrm{CD}$ and $\mathrm{UC}$, respectively [3]. Regional heterogeneity of allele frequencies among different sub-populations has been observed $[4,5,21]$, and indicates
Table 4 For genes with a significant genetic association to IBD, $C D$, or $U C$, gene expression ( $\Delta$ Ct values) was analyzed in relation to presence of inflammation, using logistic regression. $\Delta \mathrm{Ct}$ values are inversely related to gene expression values. The estimates represent the natural logarithm of the odds ratio with a negative value corresponding to increased odds for inflammation, while a positive value corresponds to decreased odds

\begin{tabular}{|c|c|c|c|c|}
\hline \multirow{2}{*}{$\begin{array}{l}\text { Gene } \\
\text { expression }\end{array}$} & \multicolumn{4}{|c|}{ Single logistic regression } \\
\hline & $p$ value & Estimate & Estimate $(95 \% \mathrm{Cl})$ & Nagelkerke $\mathrm{R}^{2}$ \\
\hline \multicolumn{5}{|c|}{$I_{B D}$; colonic biopsies ${ }^{a}$} \\
\hline F11R & 0.139 & 1.04 & $-0.34-2.43$ & 0.05 \\
\hline MAGI2 & 0.115 & 0.93 & $-0.22-2.08$ & 0.06 \\
\hline$M A G / 3$ & $1.53 \times 10^{-4}$ & 3.38 & $1.63-5.13$ & 0.52 \\
\hline PTEN & $6.72 \times 10^{-5}$ & 10.25 & $5.21-15.29$ & 0.69 \\
\hline PTPN22 & 0.005 & -1.76 & $-2.98--0.54$ & 0.22 \\
\hline TJP1 & $1.56 \times 10^{-4}$ & 4.97 & $2.39-7.55$ & 0.48 \\
\hline \multicolumn{5}{|c|}{$C D$; ileal biopsies ${ }^{b}$} \\
\hline$F 11 R$ & 0.768 & 0.34 & $-1.92-2.61$ & 0.01 \\
\hline MAGI2 & 0.065 & -2.48 & $-5.11-0.15$ & 0.35 \\
\hline$M A G / 3$ & 0.107 & 1.97 & $-0.43-4.36$ & 0.28 \\
\hline PTEN & 0.343 & 3.35 & $-3.58-10.27$ & 0.08 \\
\hline PTPN22 & 0.388 & 0.56 & $-0.71-1.82$ & 0.06 \\
\hline TJP1 & 0.230 & 2.07 & $-1.84-5.97$ & 0.09 \\
\hline \multicolumn{5}{|c|}{$C D$; colonic biopsies ${ }^{c}$} \\
\hline$F 11 R$ & 0.357 & 1.30 & $-1.47-4.06$ & 0.06 \\
\hline MAGI2 & 0.118 & 1.94 & $-0.49-4.38$ & 0.21 \\
\hline MAGI3 & 0.047 & 3.01 & $0.03-5.98$ & 0.39 \\
\hline PTEN & e & e & e & e \\
\hline PTPN22 & 0.029 & -2.82 & $-5.35--0.29$ & 0.45 \\
\hline TJP1 & 0.028 & 5.13 & $0.56-9.69$ & 0.50 \\
\hline \multicolumn{5}{|c|}{$U C_{;}$colonic biopsies $^{d}$} \\
\hline$F 11 R$ & 0.408 & 0.70 & $-0.96-2.35$ & 0.03 \\
\hline MAGI2 & 0.684 & 0.30 & $-1.16-1.77$ & 0.01 \\
\hline$M A G / 3$ & 0.003 & 3.39 & $1.19-5.59$ & 0.55 \\
\hline PTEN & 0.002 & 7.95 & $2.82-13.08$ & 0.57 \\
\hline PTPN22 & 0.048 & -1.54 & $-3.06--0.01$ & 0.18 \\
\hline TJP1 & 0.003 & 4.83 & $1.61-8.05$ & 0.47 \\
\hline
\end{tabular}

IBD inflammatory bowel disease, $C D$ Crohn's disease, UC ulcerative colitis ${ }^{a} n_{\text {non-inflamed }}=29$ and $n_{\text {inflamed }}=28 .{ }^{b} n_{\text {non-inflamed }}=11$ and $n_{\text {inflamed }}=6$. ${ }^{c} n_{\text {non-inflamed }}=12$ and $n_{\text {inflamed }}=7 .{ }^{d} n_{\text {non-inflamed }}=16$ and $n_{\text {inflamed }}=19$. ${ }^{\text {e}}$ Complete separation and thereby uncertain data (Mann-Whitney $U$-test showed decreased gene expression level in inflamed mucosa, compared to non-inflamed mucosa; $p=0.0004$ )

the importance of identifying associations in homogenous populations to increase study power [21] and to explain the genetic variance in IBD. To further clarify the role of the paracellular barrier in the etiology of IBD, a Swedish population of IBD patients and nonIBD controls was genotyped for several TJ related genes and analyzed for expression in the intestinal mucosa. 


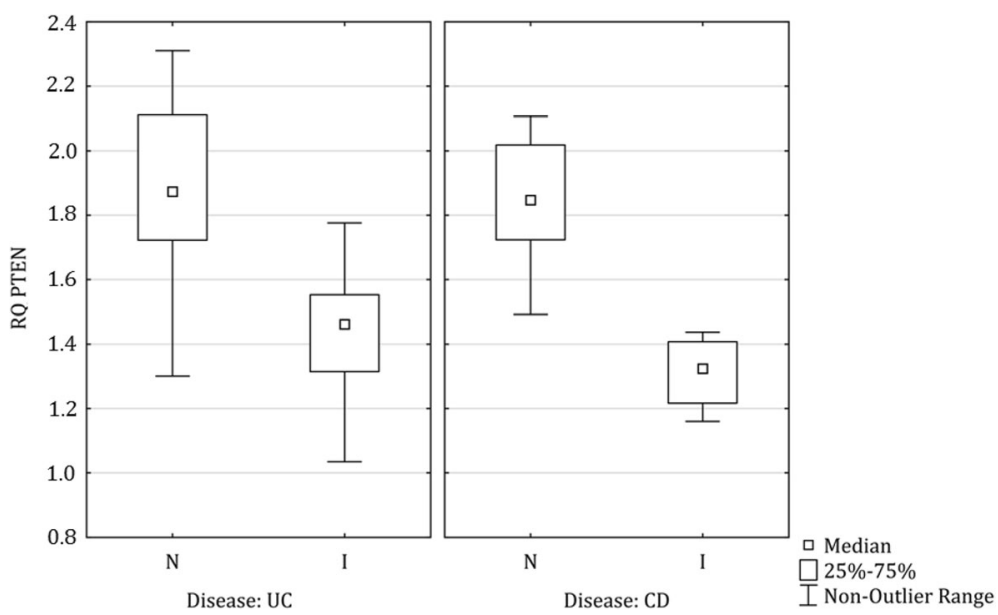

Fig 3 Box Plot illustration of PTEN expression level in colonic UC and CD mucosa. I; inflamed mucosa, N; non-inflamed mucosa

One strong association was observed between the Tallele of the MAGI3 SNP marker rs1343126 and IBD, $\mathrm{CD}$, and UC. Further, the C-allele of another MAGI3 SNP marker (rs6689879) was found to be significantly associated with UC. Presence of this marker also resulted in an increased MAGI3 expression in ileal nonIBD mucosa, which might contribute to the restriction of inflammation in UC to the colon. To the best of our knowledge we have for the first time demonstrated an association between MAGI3 and IBD. However, Jostins et al. have previously demonstrated association between this genetic region and $C D$, but primarily highlighted PTPN22 [5].

In our study, carriage of at least one IBD associated allele at rs1343126 was nominally associated to a decreased colonic expression of PTPN22 (located in the same genetic region as $M A G I 3)$ in the non-IBD subgroup. The corresponding relationship was not identified in inflamed or non-inflamed mucosa of IBD patients. In fact, all relationships between gene expression levels and the susceptibility alleles were observed in mucosal biopsies from non-inflamed, non-IBD controls. Even though the expression of several genes were affected in inflamed mucosa, it is unlikely that genotype - gene expression relationships were concealed in the IBD patients by clinical or subclinical inflammation in the non-inflamed biopsies, since these biopsies were obtained from IBD mucosa that were assessed as histopathologically normal. It is, however, possible that the investigated genes were affected by other factors (e.g. microRNA) that persist in non-inflamed IBD-mucosa [22-24].

Jostins et al. previously identified the $\mathrm{C}$-allele of rs6679677 (MAGI3-PTPN22) as a susceptibility allele of
$\mathrm{CD}$, but a protective allele with respect to UC [5], which was not confirmed by our study. Nevertheless, it is possible that our study missed a possible association due to a comparatively small sample size.

Significant associations were observed between an SNP marker in MAGI2 (rs6962966) and IBD, CD, as well as UC. Wapenaar et al. [11] previously described an association between this marker and UC. Their study revealed, however, an association to the opposite allele (G) compared to our study (A). Presently we have no explanation for this discrepancy.

In our association study we applied a significance level of $p<0.05$, and the findings were further followed up by analyzing for a genotype-gene expression correlation in a second independent cohort. However, even if we apply a more stringent $p$-value, by making a Bonferroni adjustment based on the number of analyzed genes $(n=$ 8; $p<0.006$ ), both MAGI3 (rs1343126) and MAGI2 (rs6962966) would still remain significantly associated to IBD.

One limitation of our study was that MAGI1 and MAGI3 were only investigated for SNP markers of exons and exon-intron-boundaries, due to the large size of these genes, and that the selection of SNP markers of MAGI2, MAGI3-PTPN22, PARD3, and TJP1 were based on reports in the literature.

MAGI3 was expressed to a lower level in inflamed, compared to non-inflamed, colonic mucosa from IBD and UC patients. MAGI3 is involved in suppression of the PI3K/Akt pathway due to interaction with PTEN [25], and also in the Wnt/ $\beta$-catenin pathway $[26,27]$. T cells with a high $\beta$-catenin level induce $\mathrm{T}$ cell activation and intestinal inflammation [28]. Thus, regulation of these pathways might constitute a mechanism by which 
decreased MAGI3 expression promotes intestinal inflammation. Furthermore, the PTPN22 gene expression was shown to be increased in inflamed colonic IBD mucosa, compared to non-inflamed mucosa, which corroborates previous results reported by Chen et al. [29]. The PTPN22 gene is expressed at a higher level in immune cells compared to non-immune cells [30], and further PTPN22 negatively regulates $\mathrm{T}$ cell receptor signaling [31]. In our study gene expression was determined using biopsies, and therefore we are unable to determine the exact cellular origin of the increased expression in inflamed mucosa. It may, however, be due to increased quantities of PTPN22-expressing immune cells during inflammation.

Decreased PTEN expression was observed in inflamed colonic mucosa of IBD, CD, and UC patients, compared to non-inflamed mucosa. Furthermore, there was no overlap between the PTEN expression levels in inflamed colonic mucosa of $C D$ patients, compared to non-inflamed CD mucosa, suggesting $P T E N$ as a marker for inflammatory response in colonic CD mucosa. PTEN is described to play a role in the regulation of intestinal permeability [32, 33], and is also defined as a tumor suppressor molecule inhibiting inflammatory response, cell migration, and proliferation via PI3K/Akt pathway [34, 35]. An increased level of microRNA-21 has been described in colonic biopsies from UC patients, compared to normal colonic mucosa, and expression of microRNA-21 in the Caco-2 cell line, a colonic epithelial cell model, resulted in an increased permeability [36]. The mechanism of microRNA21 has been investigated in Caco- 2 cells, and may involve an effect on paracellular permeability via the PTEN level and Akt phosphorylation [33]. Moreover, activation of PI3K/Akt pathway has been demonstrated to inhibit the differentiation of $\mathrm{T}$ cells towards regulatory $\mathrm{T}$ cells [37] and previously a decreased PTEN expression level was observed in intestinal mucosal lymphocytes in CD patients, compared to controls [38].

The decreased TJP1 expression level observed in inflamed IBD and UC mucosa, compared to non-inflamed mucosa, is consistent with a loss of ZO-1 protein (encoded by TJP1) in dextran sulfate sodium induced colitis in mice [39]. Poritz et al. further observed a correlation between loss of ZO-1 and increased paracellular permeability [39].

Gene expression was not affected by ongoing medical treatment (thiopurines, aminosalicylates, or glucocorticosteroids), irrespective of the presence of inflammation. Nevertheless, other studies have shown that infliximab, an anti-TNF-antibody, can impact the expression of genes associated with intestinal permeability [40] and mucosal cytokine expression [41]. Among our patients, too few were treated with infliximab to allow meaningful analysis with this drug.

\section{Conclusions}

In conclusion, our findings support a genetic basis for modulation of the intestinal epithelial barrier in IBD, and point to a complex regulation of $\mathrm{TJ}$ gene expression through a secondary role of inflammation. By focusing on a Swedish population and a network of interacting T) components we have identified MAGI3 as a new candidate gene for IBD.

\section{Additional files}

Additional file 1: Table S1. Single nucleotide polymorphism markers included in the study. (XLSX $16 \mathrm{~kb}$ )

Additional file 2: Table S2. Inflammatory bowel disease-phenotypes were investigated by performing case-control approach in genetic association studies. (XLSX $24 \mathrm{~kb}$ )

Additional file 3: Table S3. For genes with a significant genetic association to $I B D, C D$, or $U C$, gene expression was analyzed in relation to phenotype, using logistic regression. (DOCX 16 kb)

Additional file 4: Table S4. For genes with a significant genetic association to $\mathrm{IBD}, \mathrm{CD}$, or $\cup \mathrm{C}$, gene expression was analyzed in relation to medical treatment, using logistic regression. (DOCX $17 \mathrm{~kb}$ )

\section{Abbreviations}

CD: Crohn's disease; $C_{T}$ : Threshold cycle; Fc: Fold change; IBD: Inflammatory bowel disease; OR: Odds ratio; RQ: Relative quantification; SNP: Single nucleotide polymorphism; TJ: Tight junctions; UC: Ulcerative colitis

\section{Acknowledgments}

We would like to thank laboratory technician Lena Svensson (Department of Clinical and Experimental Medicine, Linköping University, Sweden) for excellent technical assistance and support.

\section{Funding}

This work was supported by FORSS, the Medical Research Council of South-Eastern Sweden [grant No 236541-2012 and 235131-2012], Futurum the Academy for Healthcare, Region Jönköping County [grant No FUTURUM-338631], Bengt Ihre-Fonden [grant No 2012-SLS 254491], and Karolinska Institutets Forskningsfonder [grant No 2014fobi42063]. The funding bodies had no role in study design, data collection and analysis, decision to publish, or preparation of the manuscript.

Availability of data and materials

The datasets during and/or analyzed during the current study are available from the corresponding author on reasonable request.

\section{Authors' contributions}

All authors conceived of the study; all authors designed the study; EN contributed to all laboratory work; EN contributed to data analysis and drafted the manuscript; SA provided blood samples and patient data; EN performed the statistical analysis; all authors contributed to the context of the manuscript; all authors read and approved the final manuscript.

\section{Competing interests}

The authors declare that they have no competing interests.

Consent for publication

Not applicable.

Ethics approval and consent to participate

This study was approved by the ethics committee of Linköping University (dnr. M35-07, dnr. 2011/201-31). Written and informed consent were obtained from all study participants. 


\section{Publisher's Note}

Springer Nature remains neutral with regard to jurisdictional claims in published maps and institutional affiliations.

\section{Author details}

'Department of Medicine, Karolinska Institutet, Solna, Stockholm, Sweden. ²Division of Medical Diagnostics, Region Jönköping County, Jönköping, Sweden. ${ }^{3}$ GastroCentrum, Karolinska University Hospital, Solna, Stockholm, Sweden. ${ }^{4}$ Department of Clinical and Experimental Medicine, Faculty of Health Sciences, Linköping University, Linköping, Sweden.

Received: 16 August 2016 Accepted: 10 May 2017

Published online: 25 May 2017

\section{References}

1. Peloquin JM, Nguyen DD. The microbiota and inflammatory bowel disease: insights from animal models. Anaerobe. 2013;24:102-6.

2. Loddo I, Romano C. Inflammatory Bowel Disease: Genetics, Epigenetics, and Pathogenesis. Front Immunol. 2015;6:551.

3. Ellinghaus D, Jostins L, Spain SL, Cortes A, Bethune J, Han B, et al. Analysis of five chronic inflammatory diseases identifies 27 new associations and highlights disease-specific patterns at shared loci. Nat Genet. 2016;48(5):150-8.

4. Liu JZ, van Sommeren S, Huang H, Ng SC, Alberts R, Takahashi A, et al. Association analyses identify 38 susceptibility loci for inflammatory bowel disease and highlight shared genetic risk across populations. Nat Genet. 2015;47(9):979-86.

5. Jostins L, Ripke S, Weersma RK, Duerr RH, McGovern DP, Hui KY, et al. Hostmicrobe interactions have shaped the genetic architecture of inflammatory bowel disease. Nature. 2012;491(7422):119-24.

6. John LJ, Fromm M, Schulzke JD. Epithelial barriers in intestinal inflammation. Antioxid Redox Signal. 2011;15(5):1255-70.

7. Lee $\mathrm{SH}$. Intestinal permeability regulation by tight junction: implication on inflammatory bowel diseases. Intestinal res. 2015;13(1):11-8.

8. Hollander D, Vadheim CM, Brettholz E, Petersen GM, Delahunty T, Rotter ال I. Increased intestinal permeability in patients with Crohn's disease and their relatives. A possible etiologic factor. Ann Intern Med. 1986;105(6):883-5.

9. Vetrano S, Danese S. The role of JAM-A in inflammatory bowel disease: unrevealing the ties that bind. Ann N Y Acad Sci. 2009;1165:308-13.

10. Buhner S, Buning C, Genschel J, Kling K, Herrmann D, Dignass A, et al. Genetic basis for increased intestinal permeability in families with Crohn's disease: role of CARD15 3020insC mutation? Gut. 2006;55(3):342-7.

11. Wapenaar MC, Monsuur AJ, van Bodegraven AA, Weersma RK, Bevova MR, Linskens RK, et al. Associations with tight junction genes PARD3 and MAGI2 in Dutch patients point to a common barrier defect for coeliac disease and ulcerative colitis. Gut. 2008;57(4):463-7.

12. McGovern DP, Taylor KD, Landers C, Derkowski C, Dutridge D, Dubinsky M, et al. MAGI2 genetic variation and inflammatory bowel disease. Inflamm Bowel Dis. 2009:15(1):75-83.

13. KEGG PATHWAY Database. Available at: http://www.genome.jp/kegg/pathway. html (Accessed 19 November 2015).

14. STRING. Available at: http://string-db.org/. Accessed 16 Aug 2016.

15. Szklarczyk D, Franceschini A, Wyder S, Forslund K, Heller D, Huerta-Cepas J, et al. STRING v10: protein-protein interaction networks, integrated over the tree of life. Nucleic Acids Res. 2015:43(Database issue):D447-452.

16. Rivas MA, Beaudoin M, Gardet A, Stevens C, Sharma Y, Zhang CK, et al. Deep resequencing of GWAS loci identifies independent rare variants associated with inflammatory bowel disease. Nat Genet. 2011;43(11):1066-73.

17. Gabriel SB, Schaffner SF, Nguyen H, Moore JM, Roy J, Blumenstiel B, et al. The structure of haplotype blocks in the human genome. Science. 2002; 296(5576):2225-9.

18. Söderman J, Berglind L, Almer S. Gene Expression-Genotype Analysis Implicates GSDMA, GSDMB, and LRRC3C as Contributors to Inflammatory Bowel Disease Susceptibility. Biomed Res Int. 2015;2015:834805.

19. Livak KJ, Schmittgen TD. Analysis of relative gene expression data using real-time quantitative PCR and the 2 (-Delta Delta C(T)) Method. Methods. 2001;25(4):402-8

20. Soderman J, Noren E, Christiansson M, Bragde H, Thiebaut R, Hugot JP, et al Analysis of single nucleotide polymorphisms in the region of CLDN2-MORC4 in relation to inflammatory bowel disease. World J gastroenterol : WJG. 2013; 19(30):4935-43.
21. Tian C, Gregersen PK, Seldin MF. Accounting for ancestry: population substructure and genome-wide association studies. Hum Mol Genet. 2008; 17(R2):R143-150

22. Fasseu M, Treton X, Guichard C, Pedruzzi E, Cazals-Hatem D, Richard C, et al, Identification of restricted subsets of mature microRNA abnormally expressed in inactive colonic mucosa of patients with inflammatory bowel disease. PLoS one. 2010;5(10):e13160. doi:10.1371/journal.pone.0013160.

23. Planell N, Lozano JJ, Mora-Buch R, Masamunt MC, Jimeno M, Ordas I, et al. Transcriptional analysis of the intestinal mucosa of patients with ulcerative colitis in remission reveals lasting epithelial cell alterations. Gut. 2013;62(7): 967-76.

24. Peloquin J, Goel G, Huang H, Haritunians T, Sartor R, Daly M, et al. O-002 Genes in IBD-Associated Risk Loci Demonstrate Genotype-, Tissue-, and Inflammation-Specific Patterns of Expression in Terminal lleum and Colon Mucosal Tissue. Inflamm Bowel Dis. 2016:22 Suppl 1:S1.

25. Wu Y, Dowbenko D, Spencer S, Laura R, Lee J, Gu Q, et al. Interaction of the tumor suppressor PTEN/MMAC with a PDZ domain of MAGI3, a novel membrane-associated guanylate kinase. J Biol Chem. 2000;275(28):21477-85.

26. Ma Q, Zhang $Y$, Meng $R$, Xie KM, Xiong $Y$, Lin $S$, et al. MAGI3 Suppresses Glioma Cell Proliferation via Upregulation of PTEN Expression. Biomed environ sci : BES. 2015;28(7):502-9.

27. $M a ~ Q$, Yang $Y$, Feng $D$, Zheng $S$, Meng $R$, Fa $P$, et al. MAGI3 negatively regulates Wnt/beta-catenin signaling and suppresses malignant phenotypes of glioma cells. Oncotarget. 2015;6(34):35851-65.

28. Keerthivasan S, Aghajani K, Dose M, Molinero L, Khan MW, Venkateswaran V, et al. beta-Catenin promotes colitis and colon cancer through imprinting of proinflammatory properties in T cells. Sci Transl Med. 2014;6(225):225-8.

29. Chen Z, Zhang $H$, Xia B, Wang P, Jiang $T$, Song $M$, et al. Association of PTPN22 gene (rs2488457) polymorphism with ulcerative colitis and high levels of PTPN22 mRNA in ulcerative colitis. Int J Color Dis. 2013;28(10):1351-8.

30. Arimura Y, Yagi J. Comprehensive expression profiles of genes for protein tyrosine phosphatases in immune cells. Sci Signal. 2010;3(137):rs1.

31. Sharp RC, Abdulrahim M, Naser ES, Naser SA. Genetic Variations of PTPN2 and PTPN22: Role in the Pathogenesis of Type 1 Diabetes and Crohn's Disease. Front Cell Infect Microbiol. 2015:5:95.

32. Langlois MJ, Bergeron S, Bernatchez G, Boudreau F, Saucier C, Perreault N, et al. The PTEN phosphatase controls intestinal epithelial cell polarity and barrier function: role in colorectal cancer progression. PLoS One. 2010;5(12): e15742.

33. Zhang L, Shen J, Cheng J, Fan X. MicroRNA-21 regulates intestinal epithelial tight junction permeability. Cell Biochem Funct. 2015;33(4):235-40.

34. Hu Y, Li Z, Guo L, Wang L, Zhang L, Cai X, et al. MAGl-2 Inhibits cell migration and proliferation via PTEN in human hepatocarcinoma cells. Arch Biochem Biophys. 2007:467(1):1-9.

35. Tokuhira N, Kitagishi Y, Suzuki M, Minami A, Nakanishi A, Ono Y, et al. PI3K AKT/PTEN pathway as a target for Crohn's disease therapy (Review). Int J Mol Med. 2015;35(1):10-6.

36. Yang $Y, M a Y$, Shi $C$, Chen $H$, Zhang $H$, Chen $N$, et al. Overexpression of miR-21 in patients with ulcerative colitis impairs intestinal epithelial barrier function through targeting the Rho GTPase RhoB. Biochem Biophys Res Commun. 2013;434(4):746-52.

37. Sauer S, Bruno L, Hertweck A, Finlay D, Leleu M, Spivakov M, et al. T cell receptor signaling controls Foxp3 expression via PI3K, Akt, and mTOR. Proc Natl Acad Sci U S A. 2008;105(22):7797-802

38. Long $\mathrm{SH}, \mathrm{He} \mathrm{Y}, \mathrm{Chen} \mathrm{MH}, \mathrm{Cao} \mathrm{K}$, Chen $\mathrm{YJ}$, Chen BL, et al. Activation of PI3K Akt/mTOR signaling pathway triggered by PTEN downregulation in the pathogenesis of Crohn's disease. J Dig Dis. 2013:14(12):662-9.

39. Poritz LS, Garver Kl, Green C, Fitzpatrick L, Ruggiero F, Koltun WA. Loss of the tight junction protein ZO-1 in dextran sulfate sodium induced colitis. J Surg Res. 2007;140(1):12-9.

40. Toedter G, Li K, Sague S, Ma K, Marano C, Macoritto M, et al. Genes associated with intestinal permeability in ulcerative colitis: changes in expression following infliximab therapy. Inflamm Bowel Dis. 2012;18(8):1399-410.

41. Arijs I, De Hertogh G, Machiels K, Van Steen K, Lemaire K, Schraenen A, et al. Mucosal gene expression of cell adhesion molecules, chemokines, and chemokine receptors in patients with inflammatory bowel disease before and after infliximab treatment. Am J Gastroenterol. 2011;106(4):748-61. 